\title{
Retention rates of antiepileptic drugs in glioma patients: the most appropriate outcome
}

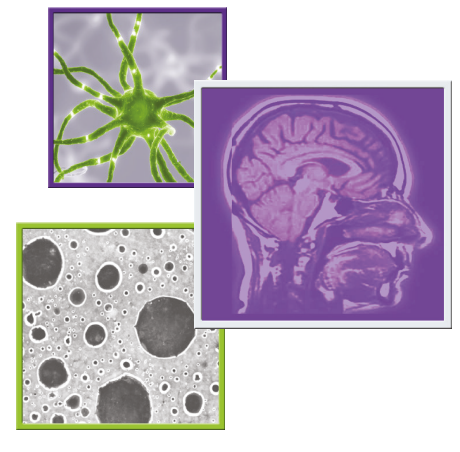

\author{
Pim B van der Meer*,1, Linda Dirven ${ }^{1,2}$, Marta Fiocco ${ }^{3,4}$, Martin JB Taphoorn ${ }^{1,2}$ \& Johan AF \\ Koekkoek ${ }^{1,2}$ \\ ${ }^{1}$ Department of Neurology, Leiden University Medical Center, Leiden, The Netherlands \\ ${ }^{2}$ Department of Neurology, Haaglanden Medical Center, The Hague, The Netherlands \\ ${ }^{3}$ Department of Biomedical Data Sciences, Medical Statistics, Leiden University Medical Center, Leiden, The Netherlands \\ ${ }^{4}$ Mathematical Institute, Leiden University, Leiden, The Netherlands \\ *Author for correspondence: Tel.: +31 71526 2192; Fax: +31 71524 8253; pbvandermeer@lumc.nl
"The retention rate is the most appropriate primary outcome to determine AED effectiveness in the glioma population because it encompasses both AED efficacy and tolerability and can be reliably estimated." "

First draft submitted: 15 April 2020; Accepted for publication: 16 April 2020; Published online: 30 June 2020

Keywords: anticonvulsants $\bullet$ antiepileptic drugs • brain tumor • glioma • lacosamide • levetiracetam • pregabalin - retention rates $\bullet$ seizures $\bullet$ treatment failure

The most common primary malignant brain tumors are gliomas, of which glioblastoma accounts for the majority [1]. Seizures are a frequently occurring presenting symptom in glioma patients and the incidence of seizures is higher in slow-growing and IDH-mutated gliomas. Preoperative seizure incidence in diffuse glioma ranges from approximately $25 \%$ in WHO (Geneva, Switzerland), grade IV glioblastoma IDH-wildtype to approximately $75 \%$, in grade II diffuse astrocytoma IDH-mutant and oligodendroglioma IDH-mutant 1p/19q codeleted patients [2]. A higher epilepsy burden is known to be associated with clinically relevant and a statistically significant decreased health-related quality of life and neurocognitive impairment [3], greater morbidity and has a negative impact on fitness to drive, employment, sexual activity and mood $[4,5]$. As optimizing seizure control is highly desired, all patients with a first seizure should be treated with antiepileptic drugs (AEDs) [6]. Treatment with AEDs can be challenging in glioma patients as several aspects need to be considered: potential interactions between AEDs and chemotherapy [6], increased occurrence of adverse effects compared with nontumoral epilepsy patients receiving AEDs [7] and pharmacoresistance, in other words, failure to achieve seizure freedom even after AED dual therapy [8], in approximately $20 \%$ of glioma patients [9]. In addition, factors associated with a higher epilepsy burden, such as neurocognitive impairment, might equally be attributed to the usage of AEDs and should be taken into account when prescribing AEDs [3]. Older enzyme inducing AEDs, such as phenytoin and phenobarbital, have been especially associated with neurocognitive deficits [3]. Nowadays, consensus exists that enzyme inducing AEDs are not preferred in glioma patients, but instead AEDs with less or no drug-to-drug interactions, such as levetiracetam, lacosamide, pregabalin and valproic acid [6]. Given the high degree of complexity of seizure management in glioma patients, AED studies in glioma patients are of high importance to guide clinicians in anticonvulsant treatment. However, to be of value, clinical outcomes need to be reliable and chosen adequately. Here, we discuss different aspects regarding measuring AED outcomes in glioma studies: the difference between efficacy and effectiveness of AED treatment; why AED retention rate seems the most appropriate primary outcome in brain tumor related epilepsy studies; how the retention rate should statistically be analyzed and current literature on AED retention rates in glioma patients. 


\section{Efficacy versus effectiveness}

According to the International League Against Epilepsy (London, UK) the primary outcome in epilepsy studies is either efficacy or effectiveness, evaluated after a minimum of 24 or 48 weeks of treatment, respectively. 'Efficacy' is defined as the ability of an AED to achieve seizure freedom. 'Effectiveness' refers to both AED efficacy and 'tolerability', with the latter encompassing the incidence, severity and impact of AED-related adverse effects, of which discontinuation of an AED due to intolerable or life-threatening adverse reactions is the most important. Effectiveness is reflected in the AED 'retention rate', defined as the percentage of patients continuing AEDs at the end of a specified period [10]. The usefulness of a drug decreases with an increasing rate of patients having to discontinue their AED due to uncontrolled seizures, intolerable adverse effects or for other reasons. Different terms exist for retention rate, including survival rate, continuation rate, discontinuation rate and treatment failure rate. Although used interchangeably, these terms may have different meanings, for example continuation and discontinuation rates are opposite concepts. Of note, retention rates not only applies to AEDs, but is an increasingly acknowledged outcome in many medical fields in which patients receive continuous pharmacological treatment, such as rheumatology and psychiatry $[11,12]$.

\section{Why retention rate is the most appropriate primary outcome}

Encompassing both AED efficacy and tolerability, the retention rate has great clinical utility. So far, seizure freedom and $\geq 50 \%$ seizure reduction are more regularly used as primary or secondary outcomes in glioma studies. However, these outcomes reflect efficacy only and because efficacy outcomes largely depend on self reports of patients, they are vulnerable to recall bias. The majority of glioma patients have neurocognitive impairment, such as impaired memory [3], likely aggravating recall bias, thus hampering reliable counting of seizures necessary for outcomes relying on seizure frequency [5]. In epilepsy drug trials patients might be instructed to make use of a seizure diary, but this is unattainable in a retrospective study design and instead researchers have to depend on seizure frequency reporting in medical electronical records, further complicating a reliable estimation of seizure frequency. The management of AED treatment in glioma patients is mostly done by either the treating neurologist or neurosurgeon and they see the glioma patient in regular follow-up visits every 3- or 6 months, depending on the tumor grade. Therefore, a change in AED regimen and the reasons for this change are generally reported adequately in medical electronical records, supporting our belief that the retention rate is the most appropriate outcome in epilepsy studies.

\section{Competing risks analysis to estimate the cumulative incidence of nonretention \& first seizure}

It is important to use the correct methodology to provide a reliable estimate of the cumulative incidence of time to nonretention and to first seizure from initiation of AED treatment. Typically, Kaplan-Meier's (KM) methodology is used in the nontumoral epilepsy population and also in two studies in the glioma population to estimate AED retention $[13,14]$ instead of crude percentages. However, this method is not the most appropriate to estimate AED retention rates [15], particularly in the glioma population.

Given the dismal prognosis of several subtypes of glioma [16], death is an important factor to take into account when calculating AED retention in this patient population. The KM-estimator is based on the assumption that censoring is noninformative, meaning that censored patients, in other words patients with incomplete information about their AED retention time, have the same probability of experiencing the event of interest as patients still at risk in the population under observation. This means that time of event and censoring time are independent. As censoring is informative, in case death occurs before the event of interest, the KM-estimator is not the appropriate methodology to estimate the time of event of interest. Indeed, death is a competing event for nonretention, as patients who have died can no longer experience nonretention (e.g. due to intolerable adverse effects) of the AED. When patients who have died are censored, the KM overestimates the probability of developing nonretention. The independent assumption is not violated when, for example, a patient moves to the other side of the country and thus drops out, while this is unrelated to the event of interest [17]. In studies estimating time to first seizure, or seizure freedom rates, in glioma patients [18], where a patients dies before the occurrence of a first seizure, KM's methodology yields to bias as discussed previously. Therefore, to estimate the cumulative incidence of nonretention or first seizure, a competing risks model with death as competing risk must be used.

Not taking into account competing risks is common. Six out of 16 (38\%) of Walraven and McAlister's randomly selected studies from prominent medical journals performed a KM analysis, overestimating the event of interest by at least $10 \%[19]$. We strongly encourage using a competing risks model to estimate the cumulative incidence function for time to nonretention or time to first seizure from initiation of AED treatment. Competing risks models 
are available in several statistical packages, including R, STATA and SAS. Easy guides for clinicians are published on how to perform competing risks analysis [20].

\section{Retention rates of AEDs in glioma studies}

Although there is a limited number of studies on AED retention rates in the glioma population, none of them have incorporated a competing risks analysis. In one multicenter randomized controlled trial, monotherapy levetiracetam was compared with monotherapy pregabalin in primary brain tumor patients, most of them diagnosed with a grade III-IV glioma. After 12 months of follow-up, levetiracetam showed a retention rate of 59\%, while pregabalin showed a retention rate of $41 \%$. About a quarter of the 52 randomized patients died during the study period and nonretention was mainly due to intolerable adverse effects [13]. In a prospective, multicenter, single-arm, observational, add-on study in 93 grade I-II glioma patients, the estimated 6 month retention rate of lacosamide was $86 \%$ [14], indicating favorable effectiveness of especially levetiracetam and lacosamide.

\section{Conclusion}

The retention rate is the most appropriate primary outcome to determine AED effectiveness in the glioma population because it encompasses both AED efficacy and tolerability and can be reliably estimated. However, currently it has been scarcely implemented in the glioma population and, when applied, the proper methodology has not been used. When calculating AED nonretention rates or time to first seizure in brain tumor patients with a limited prognosis, competing risks models should be used where death is included as a competing event.

\section{Financial \& competing interests disclosure}

The authors have no relevant affiliations or financial involvement with any organization or entity with a financial interest in or financial conflict with the subject matter or materials discussed in the manuscript. This includes employment, consultancies, honoraria, stock ownership or options, expert testimony, grants or patents received or pending, or royalties.

No writing assistance was utilized in the production of this manuscript.

\section{Open access}

This work is licensed under the Attribution-NonCommercial-NoDerivatives 4.0 Unported License. To view a copy of this license, visit http://creativecommons.org/licenses/by-nc-nd/4.0/

\section{References}

1. Ostrom QT, Gittleman H, Truitt G, Boscia A, Kruchko C, Barnholtz-Sloan JS. CBTRUS Statistical report: primary brain and other central nervous system tumors diagnosed in the USA in 2011-2015. Neuro. Oncol. 20(Suppl. 4), iv1-iv86 (2018).

2. Phan K, Ng W, Lu VM et al. Association between IDH1 and IDH2 mutations and preoperative seizures in patients with low-grade versus high-grade glioma: a systematic review and meta-analysis. World Neurosurg. 111, e539-e545 (2018).

3. Klein M, Engelberts NH, van der Ploeg HM et al. Epilepsy in low-grade gliomas: the impact on cognitive function and quality of life. Ann. Neurol. 54(4), 514-520 (2003).

4. Aaronson NK, Taphoorn MJB, Heimans JJ et al. Compromised health-related quality of life in patients with low-grade glioma. J. Clin. Oncol. 29(33), 4430-4435 (2011).

5. Avila EK, Chamberlain M, Schiff D et al. Seizure control as a new metric in assessing efficacy of tumor treatment in low-grade glioma trials. Neuro. Oncol. 19(1), 12-21 (2017).

6. Weller M, van den Bent M, Hopkins $\mathrm{K}$ et al. EANO guideline for the diagnosis and treatment of anaplastic gliomas and glioblastoma. Lancet Oncol. 15(9), e395-e403 (2014).

7. Glantz MJ, Cole BF, Forsyth PA et al. Practice parameter: anticonvulsant prophylaxis in patients with newly diagnosed brain tumors. Report of the Quality Standards Subcommittee of the American Academy of Neurology. Neurology 54(10), 1886-1893 (2000).

8. Kwan P, Arzimanoglou A, Berg AT et al. Definition of drug resistant epilepsy: consensus proposal by the ad hoc task force of the ILAE Commission on Therapeutic Strategies. Epilepsia 51(6), 1069-1077 (2010).

9. Kerkhof M, Dielemans JC, van Breemen MS et al. Effect of valproic acid on seizure control and on survival in patients with glioblastoma multiforme. Neuro. Oncol. 15(7), 961-967 (2013).

10. Glauser T, Ben-Menachem E, Bourgeois B et al. Updated ILAE evidence review of antiepileptic drug efficacy and effectiveness as initial monotherapy for epileptic seizures and syndromes. Epilepsia 54(3), 551-563 (2013).

11. Lin P-T, Wang S-H, Chi C-C. Drug survival of biologics in treating psoriasis: a meta-analysis of real-world evidence. Sci. Rep. 8 , 16068 (2018). 
12. Gartlehner G, Hansen RA, Carey TS, Lohr KN, Gaynes BN, Randolph LC. Discontinuation rates for selective serotonin reuptake inhibitors and other second-generation antidepressants in outpatients with major depressive disorder: a systematic review and meta-analysis. Int. Clin. Psychopharmacol. 20(2), 59-69 (2005).

13. Rossetti AO, Jeckelmann S, Novy J, Roth P, Weller M, Stupp R. Levetiracetam and pregabalin for antiepileptic monotherapy in patients with primary brain tumors. A Phase II randomized study. Neuro. Oncol. 16(4), 584-588 (2014).

14. Ruda R, Hellot S, De Backer M et al. A Noninterventional Study of adjunctive lacosamide therapy in patients with brain tumor-related epilepsy (S30.006). Neurology 92(Suppl. 15), S30.006 (2019).

15. van der Meer PB, Fiocco M, Koekkoek JAF, Taphoorn MJB, Dirven L. Computation of antiepileptic drug retention rates in the presence of a competing risk. Seizure 67, 82 (2019).

16. Stupp R, Mason WP, van den Bent MJ et al. Radiotherapy plus concomitant and adjuvant temozolomide for glioblastoma. N. Engl. J. Med. 352(10), 987-996 (2005).

17. Putter H, Fiocco M, Geskus RB. Tutorial in biostatistics: competing risks and multi-state models. Stat. Med. 26(11), 2389-2430 (2007).

18. Kerkhof M, Vecht CJ. Seizure characteristics and prognostic factors of gliomas. Epilepsia 54(Suppl. 9), S12-S17 (2013).

19. van Walraven C, McAlister FA. Competing risk bias was common in Kaplan-Meier risk estimates published in prominent medical journals. J. Clin. Epidemiol. 69, 170-173 (2016).

20. de Wreede LC, Fiocco M, Putter H. mstate: An R package for the analysis of competing risks and multi-state models. J. Stat. Softw. 38(7), 1-30 (2011). 